- The degree of association between a number of social, economic and behavioural risk factors and the national prevalence data for oral cancer (incidence and mortality), dental caries and destructive periodontitis was determined.

- Statistically significant associations existed for all theses diseases but were strongest for destructive periodontitis.

- These associations should be interpreted with caution, but are suggestive of the need to take them into consideration when developing health promoting oral health policies.

\title{
Oral diseases and socio-economic status (SES)
}

\author{
M.H. Hobdell, ${ }^{1}$ E. R. Oliveira, ${ }^{2}$ R. Bautista, ${ }^{3}$ N.G. Myburgh, ${ }^{4}$ R. Lalloo, ${ }^{5}$ S. Narendran ${ }^{6}$ and N. W. Johnson ${ }^{7}$
}

Objective To determine the association between social, economic and behavioural risk factors and national prevalences of: oral cancer, dental caries (12-year-olds) and destructive periodontal disease (35-44-year-olds).

Data sources Sources for the social and economic parameters were the UN Development Program; the behavioural risk factors' source was the World Health Organization, the UN Food and Agricultural Organization and the World Atlas of History. Oral diseases data came from UICC Globocan and the World Health Organization databases.

Data extraction Data were extracted by hand from official publications. Data synthesis Data were synthesized and analyzed in sequence using SPSS, Pearson's correlation coefficient and multiple regression analyses. Conclusions There is a discernable association between the three oral diseases and the variables selected, which varies in strength, being strongest for chronic destructive periodontitis and weakest for oral cancer. Dental caries lies in between. The degree to which variables account for differences in the three oral diseases between the countries studied is striking, being insignificant for oral cancer incidence, modest for oral cancer mortality, stronger for dental caries and strongest for destructive periodontal disease. Removing variables with strong colinearity with the Human Development Index has little effect on the regression coefficients.

\section{INTRODUCTION}

One of the enduring puzzles of public health is why some populations are healthier than others. The answers to such apparently

\footnotetext{
${ }^{1}$ Professor and Chair, Department of Dental Public Health and Dental Hygiene, University of Texas Health Science Center at Houston, USA. ${ }^{2}$ Senior Resident, Advanced Education in General Dentistry program, University Of Texas Health Science Center at San Antonio, USA. ${ }^{3}$ Senior dental student, Dental Branch, University of Texas Health Science Center at Houston, USA. ${ }^{4}$ Associate Professor, Department of Community Dentistry, University of the Western Cape, Cape Town, South Africa. ${ }^{5}$ Senior Lecturer, Department of Community Dentistry, University of the Western Cape, Cape Town, South Africa. ${ }^{6}$ Associate Professor, Department of Dental Public Health and Dental Hygiene, University of Texas Health Science Center at Houston, USA. ${ }^{7}$ Professor and Head of the Department of Oral and Maxillofacial Medicine and Pathology, and the WHO Collaborating Centre for Oral Cancer and Precancer. GKT Dental Institute, London UK.

${ }^{*}$ Correspondence to: Professor Martin H. Hobdell, Department of Dental Public Health and Dental Hygiene, University of Texas Health Science Center at Houston, 6516, MD Anderson Boulevard, Houston, Texas, 77030, USA.

Email:martin.h.hobdell@uth.tmc.edu
}

\section{Refereed paper}

Received 01.03.02; Accepted 06.09.02

$\odot$ British Dental Journal 2003; 194: 91-96 simple questions, although complex to formulate, are crucial in understanding oral diseases and how they might be eliminated or controlled through the development of appropriate public policies and programmes.

It was long taken for granted that people of lower socioeconomic status (SES) have worse health than people of higher SES. Few studies have searched for the reasons beyond the obvious ones related to the living conditions of those in abject poverty and such explanations are inadequate to explain the differences in health between different socioeconomic groups within affluent countries. Coronary heart disease and stroke, for example, are most common among those in lower socioeconomic groups and less common in the most affluent populations in the affluent countries. These are just two diseases that show a SES related gradient of distribution within populations. ${ }^{1}$ There are many others.

In their summary evidence on 'Inequalities in Oral Health' to the UK Independent Inquiry into Inequalities in Health, Sheiham and $\mathrm{Watt}^{2}$ state that the main causes of inequalities (in oral health) are differences in patterns of consumption of non-milk extrinsic sugars (NMES) and fluoridated toothpaste. Improvements in oral health that have occurred over the last 30 years have been largely a result of fluoride toothpaste and social, economic and environmental factors. Oral health inequalities will only be reduced through the implementation of effective and appropriate oral health promotion policy. Treatment services will never successfully tackle the underlying cause of oral disease'.

The development of healthy public policies, a cornerstone of health promotion, ${ }^{4}$ is based on the premise that there is sufficient evidence identifying many of the key social and societal variables that if improved would elevate population health.

In the same way Tarlov, ${ }^{5}$ for example, has outlined certain societal features that "would at a minimum improve the general quality of living overall, but would likely improve population health as well. These features include improved opportunities for the following: successful child development, strengthened community cohesion, enhanced self-fulfilment, increased socioeconomic well-being, and modulated hierarchical structuring.' A key question and the purpose of this paper, is 'What are the relationships between SES variables and oral health when examined from an inter-country rather than an intra-country perspective?' 
With this as the background, the present study was established specifically to investigate associations between the patterns of certain oral conditions (dental caries, chronic destructive periodontal disease and oral cancer) in different countries and certain national socioeconomic characteristics of the populations (SES indicators).

The three selected oral diseases were chosen because oral cancer, unlike most other oral conditions, has a high mortality rate, especially where there are few health services available for treatment. Dental caries was chosen because it and its sequelae have been the main concern of most modern dentists the world over. Destructive periodontal disease was chosen because its prevention and treatment provide a second major part of the workload of practicing dentists. Both dental caries and destructive periodontal diseases are highly prevalent and create much morbidity the world over: they are very expensive to treat, requiring skilled personnel and considerable amounts of professional time.

In addition there are two important behavioural risk factors associated with these three oral diseases: tobacco use is associated with oral cancer and destructive periodontal diseases ${ }^{6,7}$ and, as Sheiham and Watt op. cit. emphasize, sucrose consumption with dental caries. ${ }^{8}$

The focus of the study was the examination of the influence on the national incidence and prevalence of oral diseases of SES variables known to affect, or to be implicated in, aspects of general health and life expectancy. In no way should this study be seen, either intentionally or unintentionally, to compromise the importance of the clinical care of individuals who contract these diseases or the importance which attaches to access to oral healthcare. These matters are critical but do not, in the first instance, necessarily or uniquely control the incidence or prevalence of disease in populations. Individual healthcare is just one way in which population health may be influenced.

It is not new to examine social gradients in health within countries. ${ }^{9,10,11,12,13}$ This study, rather than focusing on such stratifications within one country, examines variations in population oral health between countries and their relationship to national SES variables, to the extent that available data permit. There have been relatively few international studies of this kind. The World Health Organization, for example, has published a number of reviews comparing oral health data between regions and nations categorized as 'industrialized' or 'developing', for example Pilot, ${ }^{14}$ but with little detailed analysis. The WHO International Collaborative Studies of Oral Health have focused on some pertinent aspects, for example, the effects of oral healthcare systems, ${ }^{15}$ but this was possible only in a relatively few mainly industrialized countries. Most other studies have also focused on different national health systems and their oral health outcomes, but not on the national SES determinants of oral health. ${ }^{16}$ Nadanovsky and Sheiham ${ }^{17}$ reported on the relative contribution of dental services to changes in caries levels of 12year-old children in 18 industrialized countries in the 1970s and early 1980s.

Marmot, ${ }^{1}$ in his review of the epidemiology of SES and health, concludes that the causes of inequalities in health within countries are similar to those between countries. With only limited international comparative data concerning SES variables and oral health, it seems premature to conclude that the same is necessarily true for oral health.

In trying to answer the general question posed by this study: 'What are the relationships between SES variables and oral health when examined from an inter-country rather than an intra-country perspective?' this paper attempts, therefore, to test the veracity of Marmot's observations with respect to oral health between countries.

\section{METHODS}

Data sources for socioeconomic status (SES):

The socioeconomic indicators used were: the Human Development Index (HDI), the Urban Population Growth (UPG), Infant Mortality Rate (IMR), Mean Years of Schooling (MYS), Under-Five Mortality Rate (UMR), Gross National Product per Capita (GNP) and the GINI Index (GI). The Gini Index is a measure of the deviation in national population income distribution from a given percentage of income being received by the same percentage of the population. The SES data were obtained from the United Nations Development Programme (UNDP). ${ }^{18}$ The Gini Index data were obtained from the World Bank. ${ }^{19}$ The number of countries for which different types of data were available varied for each separate parameter depending on the SES entity (Table1).

\begin{tabular}{lllc} 
Table 1 & & & \\
\hline SES variable & Acronym & Data year & Number of countries \\
\hline Human Development Index & HDI & 1992 & 164 \\
Infant Mortality Rate & IMF & $1988-1992$ & 127 \\
Under Five Mortality Rate & UFMR & $1988-1992$ & 127 \\
Mean Years of Schooling & MYS & 1992 & 163 \\
Urban Population Growth Rate & UPG & $1960-1992$ & 142 \\
Gross National Product & GNP & $1980-1991$ & 135 \\
Gini Index & GI & $1990-1999$ & 102
\end{tabular}

\section{Behavioural risk factors}

Behavioural risk factors data were obtained from the UN Food and Agricultural Organization, Food Balance Sheet ${ }^{20}$ (sugar) in the same way as Sreebny ${ }^{21}$ reported and the World Health Organization Tobacco or Health Report, ${ }^{2}$ and civil strife and/or instability were taken from the Atlas of World History. ${ }^{23}$ The latter variable was not measured on an ordinal scale, but was a simple categorical classification of countries. Either civil strife and/or instability were present during the selected time period or they were absent. The number of countries for which different types of data were available varied for each separate parameter depending on the aetiologic factor (Table 2).

\begin{tabular}{lccc} 
Table $\mathbf{2}$ & & & \\
\hline Aetiological variable & Acronym & Data year & Number of countries \\
\hline Mean grams sugar/day/capita & MGS & 1990 & 99 \\
$\begin{array}{l}\text { Mean number of manufactured } \\
\text { cigarettes/day adults 15 years }\end{array}$ & C/D & 1992 & 114 \\
$\begin{array}{l}\text { and over. } \\
\text { Civil strife/instability }\end{array}$ & CSI & &
\end{tabular}

\section{Data sources for oral diseases}

Oral cancer data were obtained from the UICC Globocan database. ${ }^{24}$ This is a computerized database that comes from the same sources as the Incidence of Cancer in Five Continents - the IARC. The following ICD9 codes were included in the oral cancer data abstracted: lip 140, tongue 141, mouth 143-145, oropharynx 146, hypopharynx 148, other and ill defined sites within lip, oral cavity and pharynx 149. Dental caries and periodontal disease data were obtained from the WHO Global Epidemiology Data Bank. ${ }^{25}$ The number of countries for which different types of data were available varied for each separate parameter depending on the disease entity (Table 3).

\begin{tabular}{|c|c|c|c|}
\hline Oral disease variable & Acronym & Data year & Number of countries \\
\hline $\begin{array}{l}\text { Age-standardized oral cancer } \\
\text { mortality rate by gender }\end{array}$ & MortASR & 1990 & 172 \\
\hline $\begin{array}{l}\text { Age-standardized oral cancer } \\
\text { incidence rate by gender }\end{array}$ & IncASR & 1990 & 172 \\
\hline $\begin{array}{l}\text { Dental caries: } 12 \text {-year-old, } \\
\text { decayed missing and filled teeth index }\end{array}$ & DMFT & 1989-1996 & 99 \\
\hline $\begin{array}{l}\text { Community periodontal index } \\
\text { of treatment need: } 35-44 \text {-year-olds }\end{array}$ & CPITN & 1991 & 44 \\
\hline
\end{tabular}




\begin{tabular}{|c|c|c|c|c|c|c|c|c|c|c|c|c|c|c|}
\hline \multirow{2}{*}{$\begin{array}{l}\text { OH status/ } \\
\text { SES }\end{array}$} & \multicolumn{2}{|c|}{ HDI } & \multicolumn{2}{|c|}{ MYS } & \multicolumn{2}{|c|}{ UPG } & \multicolumn{2}{|c|}{ GNP } & \multicolumn{2}{|c|}{ IMR } & \multicolumn{2}{|c|}{ UFMR } & \multicolumn{2}{|c|}{$\mathrm{Gl}$} \\
\hline & $r$ & $\mathrm{p}$ & $r$ & $p$ & $r$ & $p$ & $r$ & $\mathrm{p}$ & $r$ & $p$ & $r$ & $\mathrm{p}$ & $r$ & $\mathrm{p}$ \\
\hline \multicolumn{15}{|l|}{ MorASR } \\
\hline Male: $N=172$ & -0.16 & 0.05 & -0.16 & 0.04 & 0.14 & 0.10 & -0.17 & 0.05 & 0.16 & 0.08 & 0.15 & 0.08 & -0.12 & 0.23 \\
\hline Female: $\mathrm{N}=171$ & -0.30 & 0.00 & -0.30 & 0.00 & 0.29 & 0.00 & -0.23 & 0.00 & 0.24 & 0.01 & 0.21 & 0.02 & 0.04 & 0.66 \\
\hline \multicolumn{15}{|l|}{ IncASR } \\
\hline Male: $N=172$ & -0.01 & 0.90 & 0.00 & 0.99 & -0.02 & 0.81 & 0.00 & 0.94 & 0.03 & 0.73 & 0.03 & 0.73 & -0.11 & 0.28 \\
\hline Female: $N=171$ & -0.29 & 0.00 & -0.27 & 0.00 & 0.22 & 0.01 & -0.17 & 0.05 & 0.17 & 0.06 & 0.14 & 0.11 & 0.08 & 0.41 \\
\hline DMFT: N= 99 & 0.34 & 0.00 & 0.24 & 0.00 & -0.31 & 0.00 & -0.13 & 0.87 & -0.23 & 0.00 & -0.27 & 0.00 & 0.18 & 0.04 \\
\hline CPITN 3+4: N= 44 & -0.42 & 0.00 & -0.36 & 0.00 & 0.27 & 0.01 & -0.17 & 0.13 & 0.50 & 0.00 & 0.51 & 0.00 & 0.38 & 0.00 \\
\hline CPITN 4 & -0.50 & 0.00 & -0.46 & 0.00 & 0.38 & 0.00 & -0.28 & 0.01 & 0.51 & 0.00 & 0.54 & 0.00 & 0.48 & 0.00 \\
\hline
\end{tabular}

Table 5 Pearson's correlation coefficients for the components of the 12-year-old DMFT and SES variables

\begin{tabular}{|c|c|c|c|c|c|c|c|c|c|c|c|c|c|c|c|c|}
\hline \multirow[b]{2}{*}{$N=99$} & \multicolumn{2}{|c|}{$\mathrm{HDI}$} & \multicolumn{2}{|c|}{$\mathrm{GI}$} & \multicolumn{2}{|c|}{ GNP } & \multicolumn{2}{|c|}{ UGR } & \multicolumn{2}{|c|}{ IMR } & \multicolumn{2}{|c|}{ UFMR } & \multicolumn{2}{|c|}{ MYS } & \multicolumn{2}{|c|}{ Sugar } \\
\hline & $r$ & $p$ & $r$ & $p$ & $r$ & $p$ & $r$ & $p$ & $r$ & $p$ & $r$ & $p$ & $r$ & $p$ & $r$ & $p$ \\
\hline D & -0.18 & 0.03 & 0.37 & 0.00 & -0.41 & 0.00 & 0.13 & 0.14 & 0.11 & 0.23 & 0.08 & 0.39 & -0.25 & 0.00 & -0.00 & 0.98 \\
\hline M & 0.01 & 0.90 & -0.04 & 0.72 & -0.24 & 0.01 & -0.11 & 0.22 & -0.13 & 0.16 & -0.14 & 0.13 & -0.07 & 0.42 & -0.07 & 0.49 \\
\hline $\mathrm{F}$ & 0.67 & 0.00 & -0.38 & 0.00 & 0.51 & 0.00 & -0.65 & 0.00 & -0.59 & 0.00 & -0.56 & 0.00 & 0.77 & 0.00 & 0.63 & 0.00 \\
\hline
\end{tabular}

\begin{tabular}{|c|c|c|c|c|c|c|c|c|c|c|}
\hline \multirow[b]{3}{*}{$\mathrm{N}=$ countries } & \multicolumn{2}{|c|}{ MortASR } & \multicolumn{2}{|c|}{ IncASR } & \multicolumn{2}{|c|}{ DMFT } & \multicolumn{2}{|c|}{ CPITN 3 and 4} & \multicolumn{2}{|c|}{ CPITN 4} \\
\hline & $R^{2}$ & $\mathrm{p}$ & $\mathrm{R}^{2}$ & $\mathrm{p}$ & $\mathrm{R}^{2}$ & $p$ & $\mathrm{R}^{2}$ & $\mathrm{p}$ & $\mathrm{R}^{2}$ & $\mathrm{p}$ \\
\hline & \multicolumn{2}{|c|}{171} & \multicolumn{2}{|c|}{171} & \multicolumn{2}{|c|}{99} & \multicolumn{2}{|c|}{44} & \multicolumn{2}{|c|}{44} \\
\hline HDI & 0.06 & 0.00 & 0.01 & 0.07 & 0.12 & 0.00 & 0.18 & 0.00 & 0.25 & 0.00 \\
\hline $\mathrm{Gl}$ & 0.00 & 0.64 & 0.00 & 0.61 & 0.03 & 0.04 & 0.14 & 0.00 & 0.23 & 0.00 \\
\hline GNP & 0.03 & 0.00 & 0.00 & 0.36 & 0.00 & 0.90 & 0.03 & 0.13 & 0.08 & 0.01 \\
\hline UPG & 0.05 & 0.00 & 0.00 & 0.32 & 0.10 & 0.00 & 0.07 & 0.01 & 0.15 & 0.00 \\
\hline IMR & 0.03 & 0.00 & 0.01 & 0.24 & 0.05 & 0.00 & 0.25 & 0.00 & 0.26 & 0.00 \\
\hline UFMR & 0.03 & 0.01 & 0.00 & 0.30 & 0.07 & 0.00 & 0.25 & 0.00 & 0.29 & 0.00 \\
\hline MYS & 0.06 & 0.00 & 0.01 & 0.11 & 0.06 & 0.00 & 0.14 & 0.00 & 0.21 & 0.00 \\
\hline MGS/C/D* & 0.08 & 0.00 & 0.02 & 0.02 & 0.16 & 0.00 & 0.06 & 0.04 & 0.08 & 0.01 \\
\hline CSI & 0.001 & 0.57 & 0.00 & 0.76 & 0.00 & 0.93 & 0.06 & 0.02 & 0.05 & 0.04 \\
\hline
\end{tabular}

\section{Data analysis}

Data were analyzed in the following sequence:

- Median of the mean age standardized mortality rate and incidence rate, and median of the mean DMFT and CPITN 3 and 4 for countries grouped into low, medium and high HDI levels (using the UNDP categorization) were calculated (Figures 1-4).

- Associations between oral diseases and several SES indicators were tested using Pearson's correlation coefficient (Table 4).

- In order to explore the possible effects of greater access to dental treatment in the higher SES countries the data for the 12-yearold DMFT were separated into the component parts D, M, F, and Pearson's correlation coefficient calculated separately for each part (Table 5).

- Regression analysis was used to determine how much of the disease variation could be explained by each of the SES indicators and aetiological factors in turn (Table 6).

- In a second regression analysis the combined impact of all the SES variables and aetiological variables was explored (Table 7). Because of the considerable co-linearity between the HDI and the IMR and MYS, these variables were omitted sequentially to test the effects (Table 7).

- Significance level was set at $\alpha$ less than 0.05.

\section{RESULTS}

The median age-standardized mortality and incidence rates for oral cancer, by national HDI level are given in Figure 1. The

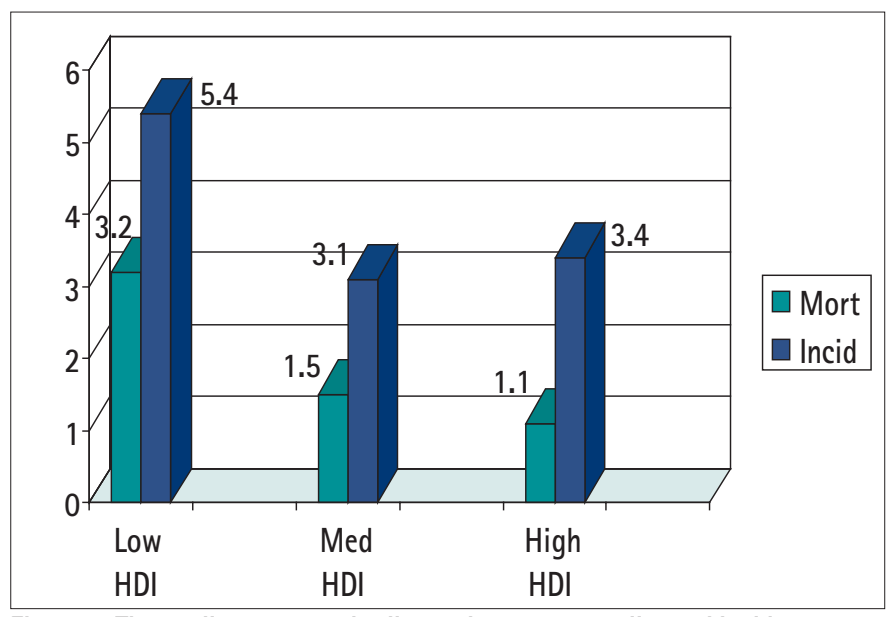

Figure 1 The median age standardize oral cancer mortality and incidence rates (male and female) for countries grouped by HDI into low medium and high using the UNDP classifications for 1992. 


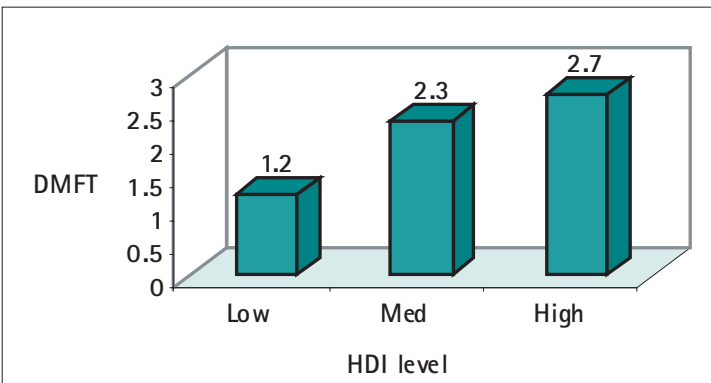

Figure 2 The median of the mean DMFT score at 12 years of age for countries grouped by HDI into low, medium and high using the UNDP classifications for 1990.

median DMFT score, at 12-years of age, by national HDI level, is shown in Figure 2. The median of the mean number of sextants scoring CPITN 3 and 4, and CPITN 4 only, by national HDI level, are given in Figure 4. The levels of association between the different oral diseases and the SES variables are given in Table 4. In Table 5 the components of the 12-year-olds DMFT are separated and the degree of association with the SES and other variables is explored. Table 6 gives the results of the regression analysis of the individual SES variables with the different oral diseases. In Table 7 all the SES variables are in the regression analysis together with the additional aetiological elements of cigarette use (for adults $>15$ years) and sugar consumption (mean number of grams per day, per capita). Table 7 also contains the regression model after adjusting for co-linearity effects of the HDI, MYS, and IMR.

\section{DISCUSSION}

This is a preliminary report of a work in progress and the findings should be seen as exploratory in this field of research rather than definitive. Data have been culled from a variety of different sources and although they are the most comprehensive so far gathered together, they are neither all of the same quality, nor as extensive as would be desirable. Nonetheless, with this strong proviso, it is believed that there are some valid points of considerable interest.

The dataset used in this study has some important limitations that arise from the fact that they were never gathered, nor intended to be used, as a single consistent whole. First, both dependent and independent variables are a mixture of cross sectional and longitudinal observations, as is common in ecological studies of this type. To what extent this limits both the usefulness of the data and the observations made from such studies is debatable. Prospective studies would likely overcome such shortcomings to some extent.

Second, because of the nature of the databases used and the comment above it was not feasible in this study to take cognizance of the dynamic nature of, for example, dental caries or oral cancer data. Future studies should attempt to do this.

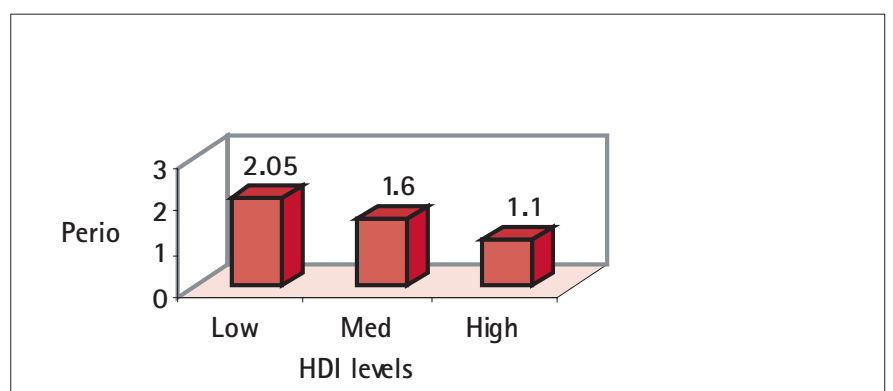

Figure 3 Periodontitis measured as median of the mean number of sextants Scoring CPITN 3+4 for countries grouped by HDI into low, medium and high using the UNDP classification for 1990.

As stated above this is an ecological study. Such studies provide the lowest level of epidemiological evidence in the search for the causes of diseases largely because the analysis is done at the macro level. The problem with ecological studies is that such studies are unable to link or match individual behaviour to individual disease experience. The issue of controlling for cofounders and colinearity of variables is another example. In this paper, for example an attempt was made to examine the effects of colinearity between the HDI, IMR and MYS, but there are undoubtedly others: tobacco and sugar consumption in intra-country studies are often highly related to SES status. ${ }^{26}$ Clearly there is much more work to be done in this area.

The work done in Britain, which was revived and stimulated by The Black Report, ${ }^{27}$ shows that despite over 50 years of the British National Health Service, differences in health status persist across social classes and geographical regions. Such differences also persist in oral health, despite the long standing universal access to dental care afforded to the UK population by the NHS. ${ }^{2}$ Whilst some of the differences between countries demonstrated by this study are undoubtedly the result of differences in access to, and the use of treatment services (eg oral cancer incidence and mortality rates; the components of the DMFT) some are unlikely to be so, not least because many of the countries examined have very limited oral healthcare services.

Many factors are involved in determining which populations become sick and which stay healthy. Specifically and importantly the social and physical environments which interact with the populations' genetic pool and the associated biology. The presence or absence of specific microorganisms and vectors; health behaviors such as dietary habits, tobacco, alcohol and drug use, and physical fitness; access to and the type of healthcare available, the organization and structure of public health services, all play a part in determining health and disease (the physical environment and the social environment, are all-important). ${ }^{28}$ The results of the present study provide further evidence that oral health policies which focus entirely on the genetic and biological determinants of health, leaving the social and societal determinants unaltered, are unlikely to be entirely successful at the level of population health.

\begin{tabular}{|c|c|c|c|c|c|c|c|c|c|c|}
\hline \multirow[b]{3}{*}{$\mathrm{N}=$ Countries } & \multicolumn{2}{|c|}{ MortASR } & \multicolumn{2}{|c|}{ IncASR } & \multicolumn{2}{|c|}{ DMFT } & \multicolumn{2}{|c|}{ CPITN 3 and 4} & \multicolumn{2}{|c|}{ CPITN 4} \\
\hline & $\mathrm{R}^{2}$ & $\mathrm{p}$ & $\mathrm{R}^{2}$ & $p$ & $\mathrm{R}^{2}$ & $p$ & $R^{2}$ & $\mathrm{p}$ & $R^{2}$ & $p$ \\
\hline & \multicolumn{2}{|c|}{171} & \multicolumn{2}{|c|}{171} & \multicolumn{2}{|c|}{99} & \multicolumn{2}{|c|}{44} & \multicolumn{2}{|c|}{44} \\
\hline Model 1 & & & & & & & & & & \\
\hline $\begin{array}{l}\text { HDI, UPG, GI, GNP, IMR, UFMR } \\
\text { MYS, MGS/C/D* and CSI }\end{array}$ & 0.15 & 0.03 & 0.08 & 0.35 & 0.41 & 0.00 & 0.45 & 0.00 & 0.53 & 0.00 \\
\hline \multicolumn{11}{|l|}{ Model 2} \\
\hline Model 1 - HDI & 0.11 & 0.07 & 0.05 & 0.64 & 0.35 & 0.00 & 0.44 & 0.00 & 0.53 & 0.00 \\
\hline \multicolumn{11}{|l|}{ Model 3} \\
\hline Model 1 - MYS and IMR & 0.13 & 0.02 & 0.06 & 0.41 & 0.31 & 0.00 & 0.41 & 0.00 & 0.52 & 0.00 \\
\hline
\end{tabular}




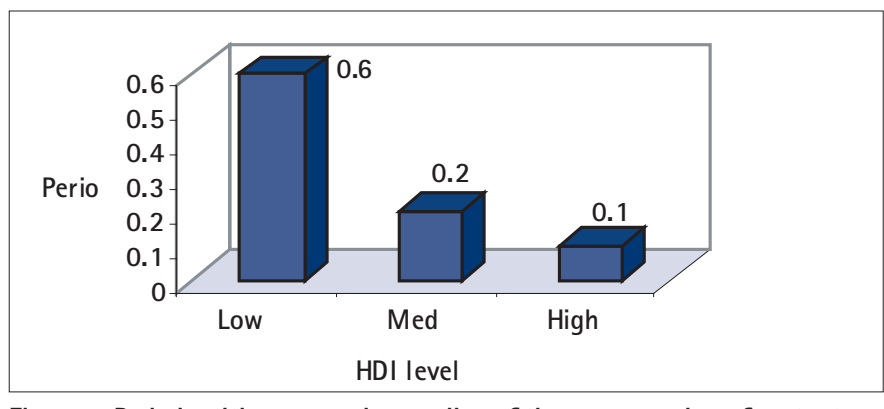

Figure 4 Periodontitis measured as median of the mean number of sextants Scoring CPITN 4 for countries grouped by HDI into low, medium and high using the UNDP classification.

Central to much contemporary research on society and health is the concept that the establishment of social hierarchies within both human and animal groups creates levels of psycho-social stress which are manifest in health gradients between those at the top of the hierarchy and those at the bottom. ${ }^{29,30}$ Currently the role that this type of stress plays in a number of specific diseases and conditions is under intensive research. In particular the quest is to define the biological pathways in which social phenomena translate into differences in levels of health. ${ }^{31,32}$

It is unfortunate that our searches failed to identify a reliable source of information for this time period concerning alcohol consumption, which has been identified as playing a marked role in the aetiology of oral cancer, particularly when combine with cigarette/tobacco use. Part of the problem is the widespread use of home or locally brewed and/or distilled alcohol, particularly, but not exclusively, in countries with lower levels of SES. Information about this alcohol consumption, unless it is bought, sold or in some way taxed does not appear in official figures, except perhaps in the relatively few household consumption surveys. One method that might be used is that reported recently by Hindle et $a .^{33}$ These authors used lung cancer and liver cirrhosis as proxy measures for cigarette and alcohol consumption.

The results of the present study clearly show a marked gradient in oral diseases between the most highly and the least socio-economically developed nations. The slope of the gradient varies for the three oral diseases examined, but is nonetheless present for all three diseases. That these SES variables alone account for approximately $50 \%$ of the differences in the prevalence of dental caries at 12 years of age and periodontitis at 35-44 years of age is noteworthy and places in perspective efforts to improve individual health by changing behavior and lifestyle as the sole focus of preventive strategies.

The slope of the dental caries gradient is the reverse of that of oral cancer and periodontitis. This may result from the fact that the DMFT index is a compound index in which treatment need D (decayed teeth) is added to treatment received M + F (missing and filled teeth). Certainly Table 5 shows some considerable differences in association with the SES variables. But it leaves no clear answer to the question as to what extent these differences reflect real differences in treatment need or whether they also reflect a philosophy which arises when restorative care becomes available. That there are differences is not surprising. Finding the answer is yet another piece of the work to be continued. In a study in which the findings of the relationship between dental caries and estimated mean annual sugar consumption in high medium and low HDI/GNP countries reported in 1982 and 1994, Hobdell, Myburgh and Lalloo, ${ }^{34}$ showed that although there was a small decrease during this period the highest level of consumption throughout was in the high GNP countries. In part this too would seem to explain the higher overall DMFT in the high HDI countries.
Of particular note in this study are the findings for periodontitis. Destructive periodontitis is well known to be strongly associated with the presence of dental plaque, smoking and alcohol consumption. It is likely that in populations with poor access to water, toothbrushes and toothpastes there is likely to be a greater accumulation of dental plaque, which would result in the strong correlation observed in this study. Above and beyond these well known and accepted aetiological factors and in the context of the present paper, it is interesting to note that since Goldhaber and Giddon $^{35}$ reported their findings in the 1960s concerning acute necrotizing ulcerative gingivitis (ANUG), the role of psychosocial stress has also been a recurring theme in the search for the aetiological factors of destructive periodontitis. ${ }^{36,37}$ The model outlined by Clarke and Hirsch ${ }^{38}$ for example, has received considerable support from a number of more recent studies. ${ }^{39-42}$ Thus the evidence supporting psychosocial stress as an aetiological factor in periodontitis is growing. The identification of possible biological pathways through which psychosocial stress may result in periodontal destruction provide circumstantial biological underpinning for the role of psychosocial stress. The findings of this present investigation only provide further food for thought on this topic but nothing more. Clearly there is much more to do in relation to psychosocial stress periodontitis, dental caries and oral cancer.

Oral cancer, at least so far as the data used in this study are concerned, seems less related than either destructive periodontitis or dental caries to the SES and aetiological variables so far as they were measured using the parameters in this study. This is surprising, as the association between oral cancer, smoking and alcohol is often credited with causing much of the disease. ${ }^{43}$ It might be that there is a stronger genetic, ${ }^{44}$ or other biological set of determinants for oral cancer ${ }^{45}$ than for periodontitis. Alternatively, it could be that there is a systematic error in the way in which these oral cancer data are recorded. Data from national cancer registers in low SES countries are notoriously weak. If, for example, data are taken from hospital (admissions) records or from a systematically biased sample of death certificates this would perhaps give rise to the results reported here.

The underlying theme of this paper that oral health is adversely affected by poor socioeconomic circumstances is not new. What is of importance is that this work, using nationally aggregated data, demonstrates clearly that it is not only individual variation that affects oral diseases prevalence but structural issues in society. It is submitted that understanding and modifying these societal structural issues might ultimately make a major contribution to reducing disparities in oral health.

\section{CONCLUSIONS}

First, there is a discernable association between the three oral diseases and the SES variables selected. The strength of the association varies. It is strongest for chronic destructive periodontitis and weakest for oral cancer. Dental caries lies in between these two.

Second, the degree to which SES variables individually account for differences in the three oral diseases between the countries studied is striking. It is insignificant for the incidence of oral cancer, modest with regard to oral cancer mortality but stronger for dental caries and strongest of all for destructive periodontal disease (CPITN 4).

Third, removing the SES variables identified as having strong co-linearity with the HDI has relatively little effect on the strength of the regression coefficients.

From these observations, chronic destructive periodontal disease would seem to be the oral disease which most clearly reflects differences in SES.

This paper raises many issues and resolves few. More work remains to be done. 
The authors are indebted to Dr Katherine Donato, Texas Institute for Society and Health, Rice University, for her valuable discussions and many suggestions concerning this paper and the presentation of the data.

MHH developed the design of the study and co-ordinated the data collection and analysis based on earlier discussions and preliminary work with NGM and RL. EO and $R B$ collated the data and assisted by $S N$ carried out the data analyses. NWJ developed the compilation of the oral cancer data and with RB structured the preliminary analyses of these data. MHH wrote the paper, which was then reviewed and revised by the other authors. All authors are guarantors.

1 Marmot M. Epidemiology of socioeconomic status and health: are determinants within countries the same as between countries? In N E Adler, M Marmot, BS McEwen Stewart J, Eds. Socioeconomic status and health in industrial nations social, psychological and biological pathways. Annals of the New York Academy of Sciences 1999; 896: 16-29.

2 Sheiham S, Watt R G. In Gordon D, Shaw M, Dorling D, Davey G, Smith Eds. Inequalities in Health. Bristol: The Policy Press, University of Bristol 1999; 240-249.

4 World Health Organization. The Ottawa Charter for Health Promotion. Health Promotion 1. iii-v, Geneva, WHO 1986.

5 Tarlov A R. Public policy frameworks for improving population health. In: Adler N E, Marmot M, McEwen B S, Stewart J, (Eds). Socioeconomic status and health in industrial nations - social, psychological and biological pathways. Annals of the New York Academy of Sciences 1999; 896: 281-293.

6 Johnson N W. Orofacial neoplasms: global epidemiology, risk factors and recommendations for research. Int Dent J 1991; 41: 365-375.

7 Elter J R, Beck J D, Slade G D Offenbach S. Etiologic models for incident periodontal attachment loss in older adults. J Clin Periodontol 1999; 26: 113-123.

8 World Health Organization. Diet, nutrition, and the prevention of chronic diseases. Geneva. Technical Report Series 1990; 797: 78-83.

9 Marmot M G, Rose G, Shipley M, Hamilton PJ S. Employment grade and coronary heart disease in British civil servants. J Epidemiol Community Health 1978; 32: 244-249.

10 Ross N A, Wolfson M C, Dunn R, Berthelot J M, Kaplan G A, Lynch J W. Relation between income inequality and mortality in Canada and in the United States: cross sectional assessment using census data and vital statistics. Br Med J 2000; 320: 898902.

11 Bosma H, Schrijvers C, Mackenbach J P. Socioeconomic inequalities in mortality and importance of perceived control: cohort study. Br Med J 1999; 319: 1469-1470.

12 Kennedy B P, Kawachi I, Glass R, Protherow-Stith D. Income distribution, socioeconomic status, and self rated health in the United States: multilevel analysis. $\mathrm{Br}$ Med J 1998; 317: 917-921.

13 Jones C M, Taylor, G O, Woods K, Whittle G, Evans D, Young P. Jarman underprivileged area scores, tooth decay and the effect of water fluoridation. Community Dent Health 1997; 14: 156-160.

14 Pilot T. The periodontal disease problem. A comparison between industrialized and developing countries. Int Dent J, 1998; 48, Supplement 1:221-232.

15 Arnljot H A, Barmes D E, Cohen L K, Hunter P B V, Ship II. Oral Health Care Systems. London: Quintessence Publishing Co Ltd, 1985.

16 Andersen R M, Marcus M, Mahshigan M. A comparative systems perspective on oral health promotion and disease prevention. In Cohen L K, Gift H C, (Eds). Oral health promotion: socio-dentalsciences in action. Copenhagen: Munksgaard International Publishers, 1995; 307-340.

17 Nadanovsky P, Sheiham A. The relative contribution of dental services to the changes in caries levels of 12 year-old children in 18 industrialized countries in the 1970 s and early 1980s. Community Dent Oral Epidemiol 1995; 23: 231-239.

18 United Nations Development Programme. Human Development Report. United Nations Development Program. Oxford: Oxford University Press 1994.

19 World Bank. Data and Statistics 1990-1999: http://www.worldbank.org.

20 United Nations Food and Agricultural Organization. Food balance sheets 1990: http://apps.fao.org.

21 Sreebny L M. Sugar vailability, sugar consumption and dental caries. Community Dent. Oral Epidemiol, 1982; 10: 1-7.

22 World Health Organization. Tobacco or Health: A global status report. Geneva, World Health Organization 1997

23 Haywood J. Atlas of World History. Oxford: Andromeda Ltd, 1997.
24 IARC-Globocan 1. Cancer Incidence and Mortality World Wide. IARC Sci Publ (CDROM) 1998.

25 Barmes D E, Sardo-Infirri J. WHO activities in oral epidemiology. Community Dent Oral Epidemio/ 1977; 5: 22-29. (Also: http://www.whocollab.odont.lu.se).

26 Coleman M P, et al. Cancer survival trends in England and Wales 1971-1995: Deprivtion and the NHS Region. London: HMSO, 1999.

27 Black D, Morris J N, Smith C Townsend P. InTownsend P, Davidson N, (Eds). Inequalities in Health: The Black Report; The Health Divide. London: Penguin Books Ltd 1992.

28 Kaplan G A. Part III Summary: What is the role of the social environment in understanding inequalities in health? In Adler N E, Marmot M, McEwen B S, Stewart J, (Eds).Socioeconomic status and health in industrial nations. New York Academy of Sciences 1999; 896: 116-119.

29 Wilkinson R G. Health, hierarchy, and social anxiety. In Adler N E, Marmot M, McEwen B S, Stewart J, (Eds).Socioeconomic status and health in industrial nations - social, psychological and biological pathways. Annals of the New York Academy of Sciences 1999; 896: 48-63.

30 Manuck S B, Kaplan J R, Adams M R, Clarkson T B. Studies of psychosocial influences on coronary artery atherosclerosis in cynomolgus monkeys. Health Psychol 1995; 113-124.

31 Francis D D, Champagne FA, Liu D, Meaney M J. Maternal care, gene expression and the development of individual differences in stress reactivity. In Adler N E, Marmot M, McEwen B S, Stewart J, (Eds). Socioeconomic status and health in industrial nations social, psychological and biological pathways. Annals of the New York Academy of Science 1999; 896: 66-84.

32 McEwen BS, Seeman T. Protective and damaging effects of mediators of stress. Elaborating and testing concepts of allostasis and allostatic load. In Adler N E, Marmot M, McEwen B S, Stewart J (Eds).Socioeconomic status and health in industrial nations - social, psychological and biological pathways. Annals of the New York Academy of Sciences, 1999; 896: 30-47.

33 Hindle I, Downer M C, Moles D R, Speight P M. Is alcohol responsible for more intraoral cancer? Oral Oncology; 2000: 328-331.

34 Hobdell M H, Myburgh N G, Lalloo R. The human development index and per capita gross national product as predictors of dental caries prevalence in industrialized and industrializing countries. In Adler N E, Marmot M, McEwen B S, Stewart J (Eds) Socioeconomic status and health in industrial nations - social, psychological and biological pathways. Annals of the New York Academy of Sciences, 1999; 896: 329 331.

35 Goldhaber P, Giddon D B. Present concepts concerning the etiology and treatment of acute necrotizing ulcerative gingivitis. Int Dent J 1963; 14: 468-496.

36 Johnson N W, Griffiths J M, Wilton J A M, Maiden M FJ, Curtis M A, Gillett I R, Wilson D T, Sterne J A C. Detection of high-risk groups and individuals for periodontal diseases. J Clin Periodontol 1988; 15: 276-282.

37 Wilton J M A, Griffiths J M, Curtis M A, Maiden M FJ, Gillett I R, Wilson D T, Sterne J A C, Johnson N W. Detection of high-risk groups and individuals for periodontal diseases -systemic predisposition and markers of general health. J Clin Periodonto/ 1988; 17 339-346.

38 Clarke N G, Hirsch R S. Personal risk factors for generalized periodontitis. J Clin Periodontol 1995; 22: 136-145.

39 Axtelius B, Edwardsson S, Theodorsson E, Svensater G, Attstrom R. Presence of cortisol in gingival crevicular fluid: A pilot study. J Clin Periodontol 1998; 25: 929 932.

40 Breivik T, Thrane PS, Gjermo P, Opstad P K. Glucocorticoid receptor antagonist RU 486 treatment reduces periodontitis in Fischer 344 rats. J Periodontal Res 2000; 35: 285 290.

41 Genco R J, Ho A W, Kopman J, Grossi S G, Dunford R G, Tedesco L A. Models to evaluate the role of stress in periodontal disease. Ann Periodontology 1998; 3: 288302.

42 Axtelius B, Soderfelt, B, Nilsson A, Edwardsson S, Attstrom R. Therapy-resistant periodontitis. Psychosocial characteristics. J Clin Periodontol 1998; 25: 482-491.

43 Johnson N W. Tobacco use and oral cancer: A global perspective. J Dent Ed 2001; 65: 328-339.

44 Jefferies $\mathrm{S}$, Eeles $\mathrm{R}$, Goldar $\mathrm{D}$. The role of genetic factors in predisposition to squamous cell carcinoma of the head and neck. Br J Cancer 1999; 79: 865-867.

45 Johnson N W. Head and neck cancer: epidemiology and aetiology of premalignant lesions. In The Oxford Textbook of Oncology. Oxford: Oxford University Press, 2002. 